\title{
Économie et politique éducative en Amérique latine
}

Economy and educational policies in Latin America

Economia y políticas educatives en America latina

Robert W. McMeekin

Traducteur : Jean-Paul Gratias

\section{OpenEdition}

\section{Journals}

Édition électronique

URL : http://journals.openedition.org/ries/2979

DOI : $10.4000 /$ ries.2979

ISSN : 2261-4265

Éditeur

Centre international d'études pédagogiques

\section{Édition imprimée}

Date de publication : 1 juin 1999

Pagination : 39-52

ISSN : 1254-4590

\section{Référence électronique}

Robert W. McMeekin, «Économie et politique éducative en Amérique latine », Revue internationale d'éducation de Sèvres [En ligne], 22 | 1999, mis en ligne le 13 mai 2013, consulté le 03 mai 2019. URL : http://journals.openedition.org/ries/2979; DOI : 10.4000/ries.2979

Ce document a été généré automatiquement le 3 mai 2019.

(c) Tous droits réservés 


\title{
Économie et politique éducative en Amérique latine
}

\author{
Economy and educational policies in Latin America \\ Economia y políticas educatives en America latina
}

\section{Robert W. McMeekin}

Traduction : Jean-Paul Gratias

1 Cet article se propose d'examiner de quelle façon l'utilisation de l'analyse économique peut fournir des points de repères et une orientation générale à une politique éducative. Cette étude se limitera à la région globalement définie par l'appellation d'Amérique latine. Cependant, certains exemples cités mettront en avant l'expérience menée au Chili. Les renseignements récents dont nous disposons sur ce sujet sont beaucoup plus nombreux dans ce cas que pour d'autres pays. D'autre part, le Chili est l'un des pays les plus avancés d'Amérique latine en ce qui concerne l'usage qu'il fait des informations et de l'analyse pour élaborer ses orientations.

2 Si l'on considère la région dans son ensemble, les instances éducatives n'ont pas habituellement recours à l'analyse économique pour élaborer leurs politiques. Ceci est vrai bien que l'Amérique latine compte de nombreux économistes et politologues compétents, souvent formés dans les universités des pays industrialisés et que les décisionnaires soient plus sensibles au poids des considérations économiques dans leurs orientations. Même si certains signes montrent que l'élaboration des politiques éducatives commence à intégrer la dimension économique, il reste beaucoup à faire dans ce domaine. Comment expliquer que l'économie soit si peu prise en compte? Les raisons sont multiples.

\section{L'absence de données}

3 Une étude récente des statistiques et des indicateurs concernant l'éducation dans la région de l'Amérique latine et des Caraïbes (ALC) a montré que les services statistiques 
des ministères de l'Éducation ont tendance à privilégier les informations venant du système scolaire et des établissements, en ignorant les données économiques, même si celles-ci sont disponibles au sein du ministère ${ }^{1}$. La même étude a mis en évidence un manque criant d'informations sur les résultats obtenus par le système éducatif. Des efforts ont été faits pour compenser ce manque d'informations. Le système régional d'information de l'UNESCO à Santiago (SIRI) a publié une série de documents intitulée L'État de l'éducation en Amérique latine et dans les Caraibes qui comprend un corpus important de statistiques et d'indicateurs pour les années 1980 à 1994². Lancé plus récemment, le projet pilote commun à l'UNESCO et à l'OCDE sur les indicateurs de l'éducation à l'échelle mondiale, financé par la Banque mondiale, inclut cinq pays d'Amérique latine: l'Argentine, le Brésil, le Chili, le Paraguay et l'Uruguay. Comme le Mexique est membre de l'OCDE, cela signifie que six des pays les plus importants de la région possèdent les données de base nécessaires au calcul des excellents indicateurs financiers de l'OCDE pour l'éducation. Les données concernant ces pays apparaissent dans la publication de l'OCDE, Education at a Glance, pour l'année $1998^{34}$. Au Sommet des Amériques de 1998, l'un des thèmes choisis pour être abordés et traités en priorité était le suivant : comment améliorer à la fois l'évaluation du système éducatif et la collecte des informations nécessaires à la conception des réformes? Le Brésil et le Chili sont les pays chargés de mettre en œuvre les mesures qui seront définies dans ces domaines. Nous reviendrons ultérieurement sur cette question de l'absence de données.

\section{La pénurie d'analystes compétents}

4 Même si, comme cela a été mentionné précédemment, il existe aujourd'hui dans la région ALC un véritable contingent d'économistes formés aux problèmes de l'éducation, rares parmi ces spécialistes sont ceux qui travaillent comme cadres ou comme conseillers dans les ministères de l'Éducation. La plupart d'entre eux se trouvent dans les universités, les instituts de recherche et les ONG, ou encore dans le secteur privé. Entre autres institutions de prestige, citons: au Mexique, le Centre d'études et de recherche en économie ; au Brésil, la Fondation Vargas; en Argentine, l'Institut Di Tella; au Chili, le Centre de recherche et de développement en éducation, le Programme interdisciplinaire de recherche éducative et le Centre d'économie appliquée de l'université du Chili, pour ne nommer que les plus connus. Il y a eu, également, une importante fuite de talents vers les organisations internationales. On trouve, évidemment, des spécialistes de haut niveau dans les ministères, mais les salaires modestes du secteur public et la rotation rapide $d u$ personnel de ces ministères (il en sera question plus bas) expliquent qu'ils ne restent pas longtemps en poste.

\section{L'absence de demande pour une analyse économique}

5 Même si les compétences existent, il se peut que les responsables chargés de définir les orientations n'éprouvent pas le besoin d'employer les outils économiques dans la conception et le développement des politiques éducatives. Bien que ce besoin soit de plus en plus reconnu dans certains pays, il n'est pas très répandu, ni très homogène. Quels sont les problèmes? Parmi eux, l'absence d'une «culture de l'information ». La plupart des responsables ont tendance à s'inspirer de considérations politiques, de la sagesse traditionnelle ou d'autres sources d'idées lorsqu'ils débattent des orientations futures. Le 
coût d'un programme ou d'une réforme, le fait qu'elle soit ou non financièrement réalisable, qu'elle rapporte ou non des avantages substantiels au regard des investissements consentis, voilà des questions qui ne sont pas souvent posées.

6 À cet état de fait s'ajoute le facteur, déjà mentionné, du renouvellement constant du personnel. Même au Chili, où le même parti est au pouvoir depuis 1990, soit deux législatures successives, il y a eu cinq ministres de l'éducation en un peu plus de huit ans, ce qui représente une présence à ce poste d'une vingtaine de mois en moyenne. Quand un ministre change, l'équipe entière de ses conseillers change aussi. C'est dans ce groupe de conseillers jouissant de la confiance du ministre que l'on trouve les décisionnaires capables de mettre sur pied une politique éducative. Cela signifie que, même dans le cas où un poste de conseiller revient à une personne compétente, qui porte sur les problèmes un regard d'économiste parce qu'elle est formée pour cela, cette personne ne reste pas à ce poste très longtemps.

7 Bien que l'on n'ait pas fréquemment recours à l'analyse économique pour orienter une politique éducative, une tendance certaine, bien que modeste, se dessine chez les décisionnaires du secteur éducatif vers une plus grande prise en compte des facteurs économiques. On a même constaté, dans quelques cas précis, une utilisation spécifique de l'analyse afin d'aider à l'élaboration de plans, de programmes et de projets.

\section{Analyse économique et élaboration d'une politique éducative}

8 Quelques remarques sur l'état de l'éducation et de son financement aideront à situer cette discussion dans son contexte. La décennie écoulée a été une période de rétablissement après les crises qui ont secoué, dans les années quatre-vingt, les pays d'Amérique latine et leurs secteurs éducatifs. Bien que les ressources disponibles pour l'éducation ne soient abondantes dans aucun des pays de la région, les dépenses par élève exprimées en pourcentage du PIB ont augmenté, comme l'ont fait, dans la plupart des cas, les traitements des enseignants. Aujourd'hui, on peut se permettre de réfléchir à la façon dont les ressources seront distribuées, au lieu de chercher désespérément les fonds nécessaires pour que le système continue de fonctionner.

9 Le contenu des programmes et projets éducatifs, délaissant les investissements destinés à favoriser l'accès à la scolarisation, s'efforce maintenant d'améliorer la qualité de l'enseignement dispensé et d'en assurer une distribution équitable. Ces changements apparaissent en évidence dans la vague de réformes éducatives qui se manifeste dans de nombreux pays de la région. Parmi les plus connues des réformes nationales, citons celles de l'Argentine, de la Bolivie, du Chili et de la Colombie, mais d'autres pays ont entrepris des changements significatifs. En plus de diverses améliorations en termes de qualité et d'équité, ces réformes visent souvent à décentraliser la direction du système éducatif ; à augmenter l'autonomie des institutions éducatives; à confier la gestion de certaines ressources aux établissements scolaires; à faire davantage participer les parents d'élèves ; à faire participer tous les membres de la communauté éducative à l'élaboration des projets et aux prises de décisions ; à renforcer le statut des chefs d'établissements ; à encourager l'innovation; à mettre en place des structures qui imposent une obligation de résultats. D'un point de vue économique, nombre de ces éléments de réforme sont judicieux ; toutefois, à notre sens, ils ont été conçus non pas sur la base d'une analyse 
économique, mais plutôt en puisant dans les résultats des recherches et des expérimentations relevant des domaines de la pédagogie et de la gestion du secteur éducatif.

10 Toute proposition de réforme de l'enseignement soulève le problème de son financement. En partant de ce principe, le Programme pour la promotion d'une réforme de l'éducation en Amérique latine (PREAL) du Dialogue interaméricain a patronné un séminaire international sur le financement de l'éducation à Bogota en juillet 1997. Le compte rendu de ce séminaire fut publié par l'UNESCO à Santiago en juin $1998^{4}$. La préoccupation majeure du PREAL étant de réformer l'enseignement, les contributions des participants traitent des moyens par lesquels les pays peuvent financer leurs réformes, souvent ambitieuses. Elles s'appuient sur diverses expériences, certaines menées hors de l'Amérique latine, d'autres qui ont eu pour cadre le Chili, le Mexique et le Pérou. L'un des articles traite du financement de l'enseignement par le secteur privé. À deux exceptions près - Stephen Barro et Alain Mingat - les auteurs sont tous latino-américains et ils représentent bien la génération d'économistes compétents, à la formation solide, que l'on trouve actuellement en Amérique latine. À un moment ou un autre de leur carrière, la plupart d'entre eux ont travaillé dans un ministère de l'Éducation ou lui ont servi de conseiller, mais à leurs yeux, leur poste le plus important est celui qu'ils occupent dans une université, une ONG qui se consacre à la recherche ou, dans certains cas, un organisme ou un institut de recherche international.

11 Quelles sont les questions primordiales d'une politique éducative auxquelles l'économie peut apporter un éclairage nouveau? Parmi les plus importantes, citons le coût du programme ou de l'orientation envisagés; les profits (en particulier, quels profits financiers) espérés de l'investissement consenti; le financement du programme et ses retombées (questions d'équité et d'incidence); la validité de l'investissement envisagé pour la réalisation des objectifs éducatifs (est-il rentable? Représente-t-il la meilleure solution possible ?) ; l'optimisation du montant des dépenses en matière d'éducation, leur utilisation efficace, leur ventilation adéquate entre les différents postes budgétaires et sous-secteurs; enfin, l'amélioration du rendement du système éducatif par des mesures incitatives

12 Au cours des inévitables rencontres entre les décisionnaires du secteur éducatif et les représentants des ministères qui allouent les ressources (ministères des Finances, du Trésor, de la Planification), le coût du programme proposé est immanquablement l'objet d'un examen scrupuleux. Les capacités d'analyse des bailleurs de fonds et les informations d'ordre économique dont ils disposent sont presque toujours supérieures à celles des auteurs du projet proposé, mais ceux-ci doivent être capables de répondre à la question : combien cela va-t-il coûter ? Et, souvent, à la question concomitante : de quelle façon espérez-vous économiser de l'argent pour compenser !a dépense prévue? À ce niveau, on constate que la capacité des responsables du ministère à donner les réponses nécessaires est de plus en plus en adéquation avec ce que l'on attend d'eux. Les réponses données peuvent parfois être volontairement faussées, en ce sens que les dépenses annoncées sont sous-évaluées; mais, si tel n'était pas le cas, il n'y aurait jamais de nouvelles réformes.

13 C'est à la phase suivante, plus complexe, des discussions, lorsqu'il s'agit d'analyser et de comparer les profits, les rendements, et les implications (du point de vue de l'efficacité et de l'équité) de l'investissement envisagé, que les faiblesses apparaissent. Ces faiblesses sont tout autant perceptibles du côté des organismes bailleurs de fonds que des instances 
éducatives. Ceci est, en partie, une conséquence supplémentaire du manque d'informations : si on ne dispose d'aucune donnée ou de données insuffisantes, soit sur les performances du système éducatif (des résultats de tests fiables), soit sur l'état du marché du travail (les taux de chômage et de rémunération), on ne peut pas procéder à de telles analyses. Mais une part importante de la raison pour laquelle les politiques éducatives ne reposent pas sur une analyse saine des coûts, du rendement, de l'efficacité et de l'équité du système se trouve dans les problèmes évoqués précédemment : le manque de demande pour ce genre d'analyse de la part des décisionnaires en charge des réformes et la pénurie d'analystes compétents aux postes où ils pourraient conseiller lesdits décisionnaires.

Certains signes montrent cependant que l'on donne de plus en plus d'importance aux facteurs économiques pendant l'élaboration des politiques éducatives. Le Chili et le Paraguay nous en fournissent deux exemples. Dans le premier de ces deux pays, une unité d'études, sous la responsabilité du directeur de la planification de l'enseignement, rassemble à présent l'ancienne unité de statistiques et une petite équipe d'analystes des stratégies chargée d'appliquer au développement et à la supervision de la réforme éducative chilienne diverses méthodes d'analyse, économiques ou autres. Quant au Paraguay, il a constitué une unité d'analyse des orientations avec l'aide technique de l'Institut Harvard pour le développement international. Il existe peut-être d'autres exemples inconnus de nous. Dans toute la région, on note une tendance de plus en plus affirmée de la part de certains responsables du secteur éducatif à élaborer leurs orientations futures en s'inspirant de ces ressources que constituent les analyses économiques et les avis des experts.

Dans ce panorama général du recours à l'analyse économique permettant de guider les politiques éducatives, il faut prendre en compte le rôle des organisations internationales. Ces entités fondent sur l'analyse économique, au moins en partie, leurs décisions concernant les projets de prêts ou de subventions. Elles ont joué un rôle central dans le développement de l'analyse du taux de rendement dans le secteur éducatif, l'un des fondements de l'économie de l'éducation ${ }^{5}$. L'un des résultats, important, de cette démarche fut de convaincre les banques internationales, particulièrement la Banque mondiale, que l'éducation constitue un investissement économique viable. L'analyse des taux de rendement a aussi permis de répondre à l'éternel problème des choix en matière de politique éducative: c'est en investissant dans l'enseignement primaire que l'on obtient les meilleurs rendements. D'autres travaux, comprenant des études de base sur les coûts et le rapport coût/faisabilité, ainsi que des tentatives pour résoudre la totalité des problèmes cités plus haut, ont permis de cerner et d'évaluer divers projets d'aide (à des degrés variables, en fonction des données disponibles). De plus, les banques internationales et la commission économique pour la région ALC poursuivent des travaux de pointe, fondés sur la recherche, concernant l'économie de l'éducation en Amérique latine, comme il en existe pour d'autres régions du globe. Ce corpus d'études et de publications joue un rôle fondamental quand il s'agit de documenter et d'orienter les débats et les décisions en matière de politique éducative. Il fournit aussi des exemples, à la fois aux «consommateurs » et aux acteurs de l'économie de l'éducation, des analyses qui doivent être effectuées et des informations directement exploitables que ces analyses peuvent fournir. 


\section{L'économie et la mise en place d'une politique éducative}

Quelle relation existe-t-il entre l'analyse économique et l'implémentation des politiques sectorielles? Alors que le lien entre l'économie et la formulation des orientations est simple et direct, le rôle de l'économie dans la mise en œuvre de ces orientations est beaucoup moins clair. Il est possible, cependant, d'énoncer quelques généralisations.

\section{Absence de continuité des personnels}

17 Pendant l'expertise de ses propres projets éducatifs, la Banque mondiale a découvert que les problèmes de mise en œuvre sont souvent dus au fait que l'équipe qui élabore un projet est rarement chargée de le mener à bien. Dans une certaine mesure, ce hiatus est inévitable, car le privilège relatif des économistes et des conseillers est de concevoir et de planifier plutôt que d'exécuter les tâches, essentiellement administratives, liées à la mise en œuvre. Le problème de la rotation du personnel restreint d'autant plus la continuité entre les concepteurs et les exécutants. D'une manière générale, cependant, l'économie joue un rôle très réduit pendant la phase d'implémentation. Les gestionnaires n'ont pas pour habitude, au cours du processus de mise en œuvre, de disposer ni d'utiliser de renseignements fournis par des commissions de surveillance ou des expertises proposant une marche à suivre ou un changement de cap.

\section{Résistance au changement}

18 La mise en place d'un nouveau programme au sein d'une organisation se heurte généralement au problème de la résistance au changement. C'est une réaction humaine normale, associée aux inquiétudes que suscite l'inconnu et à l'inconfort que provoque la nécessité de modifier des routines installées et des relations bien établies. Les spécialistes qui ont étudié le phénomène ont découvert que le remède consiste à impliquer dans la conception du projet, dès le départ, tous les acteurs concernés; à obtenir un soutien politique au plus haut niveau; à présenter clairement les objectifs du changement, sa nécessité et les bénéfices que l'on peut en tirer (à « vendre » le programme) ; à procurer au personnel une formation adaptée, à donner aux participants d'un projet l'occasion de formuler leurs inquiétudes et leurs critiques, et à prendre d'autres mesures du même type ${ }^{6}$. À ce jour, ces facteurs n'ont guère mobilisé l'attention des économistes.

\section{Centralisation}

Un problème connexe, que d'aucuns appelleraient un cas particulier de résistance au changement, est la forte tendance des dirigeants de n'importe quelle organisation à conserver le pouvoir et l'autorité qu'ils possèdent ceteris pari-bus. La vague actuelle de réformes et de programmes du secteur éducatif encourage la décentralisation de l'autorité et des responsabilités et l'une des difficultés de mise en œuvre des réformes tient à ce que les bureaucrates du ministère central cherchent des moyens de bloquer ou de minimiser les changements qui leur feraient perdre une partie de leurs pouvoirs. 


\section{Relations avec les ministères « payeurs »} mise en place de leurs programmes et projets est la pénurie des fonds partenaires. Si les gouvernements ne peuvent continuer à fournir leur part de financement du projet (qui inclut souvent les coûts de fonctionnement, en augmentation régulière, des nouveaux programmes), les autorités qui prêtent ou qui accordent les fonds cesseront leurs versements et le projet ne verra jamais le jour. Lorsque cela se produit, le coût de l'implémentation augmente et le projet tarde d'autant à porter ses fruits; en d'autres termes, il devient onéreux. Comme les ministères de l'Éducation et leurs équipes de mise en œuvre des projets ne comprennent généralement pas d'économistes capables de discuter sur un pied d'égalité avec leurs homologues des ministères des Finances ou du Trésor, ils ne peuvent présenter d'arguments convaincants pour démontrer qu'il est coûteux de bloquer les fonds nécessaires au partenariat. Le problème est aggravé par le fait que les responsables des finances ou du trésor ont tendance à considérer le secteur éducatif non comme un investissement, mais comme un poste budgétaire à fonds perdus.

\section{Manque de gestionnaires qualifiés}

Non seulement les ministères de l'Éducation connaissent une pénurie d'économistes, mais ils ont tendance à manquer de gestionnaires au fait des techniques de la maîtrise de projet, du re-engineering ou de l'implémentation des changements. On déplore aussi leur méconnaissance des avancées et des tendances récentes dans la gestion du secteur public telles que les réformes administratives instituées en Nouvelle-Zélande, et qui ont suscité un grand intérêt ${ }^{7}$.

\section{Expertise économique de la politique éducative}

Qui procède aux expertises des politiques éducatives? Est-ce le ministère de l'Éducation lui-même ou quelque autre entité ? Les cas où les ministères entreprennent de le faire eux-mêmes sont rares. En plus des problèmes mentionnés dans les chapitres précédents, on constate toujours une résistance à l'évaluation de la part de ceux qui doivent en faire l'objet. Il y a, de plus, un problème de vision à court terme (vers le passé comme vers l'avenir) de la part des officiels associés à toute administration politique donnée. Les responsables nommés par le pouvoir ont tendance à ignorer ou à déprécier tout ce qui a été fait avant leur (brève) présence au poste qu'ils occupent, plutôt que d'étudier les orientations prises par les régimes précédents pour en tirer les leçons qu'elles contiennent. On trouve parfois des tentatives sérieuses d'évaluation formative, qui peuvent mener à une réactualisation ou à une modification des choix en fonction de l'évolution des circonstances. En 1997, la Bolivie a procédé à une expertise de sa réforme éducative, encore qu'elle l'ait fait sous la forte pression des organisations internationales qui finançaient cette réforme.

Si des évaluations sommatives doivent être effectuées, elles le sont généralement par les ministères qui allouent les ressources, parce qu'ils ont intérêt à connaître le résultat des orientations prises. Sinon, les expertises sont faites par des organismes de recherche (universités ou $\mathrm{ONG}$ ) sous la forme de travaux de recherche dans le domaine de 
l'éducation. D'autre part, des organisations internationales telles que les banques de développement et la commission économique pour la région ALC pratiquent deux formes d'expertise : elles évaluent les projets spécifiques qu'elles ont financés et elles mènent des études extrêmement fouillées qui les aident à formuler leurs orientations et leurs priorités propres. Dans la première catégorie, les «rapports sur l'avancement des projets " se concentrent plutôt sur les objectifs spécifiés dans le projet d'origine pour vérifier s'ils ont été atteints. Ils ont tendance à être plus descriptifs qu'analytiques. Les recherches spécifiques réalisées ou commandées par les organisations internationales, en revanche, se caractérisent par un degré d'analyse extrêmement élevé.

Les deux catégories d'expertises sont bridées par une pénurie générale d'informations concernant les performances du système éducatif en Amérique latine. Contrairement aux pays du Commonwealth britannique, par exemple, les pays d'Amérique latine n'ont pas une expérience approfondie de l'évaluation de leurs élèves et n'ont commencé que récemment à introduire des procédures standardisées de tests de niveau. Ces nouveaux protocoles sont, dans la majorité des cas, une évaluation par échantillonnage du niveau scolaire, qui donne des indications sur le fonctionnement du système éducatif dans son ensemble, mais ne récolte pas d'informations, individuellement, sur tous les élèves. Le Chili possède un Sistema de Medición de la Calidad de la Educación (SIMCE) dans lequel tous les élèves sont évalués aux niveaux primaire et, récemment, secondaire. Les résultats de tests par échantillonnage peuvent être très utiles pour analyser les effets d'une réforme. Les tests qui concernent cent pour cent de la population scolaire sont exploitables dans des procédures d'analyse aux finalités beaucoup plus diverses.

Une avancée importante dans le domaine de l'évaluation est la fondation du Laboratorio Latinoamericano de Evaluación de la Calidad de la Educación. Basé à l'office régional de l'UNESCO pour l'éducation, jouissant d'une aide financière complémentaire de la Fondation Ford et de la Banque de développement interaméricaine, le laboratoire a coordonné une évaluation coordonnée dans treize pays d'Amérique latine. Il a récemment fait paraitre sa première publication majeure présentant les résultats de tests par échantillonnage en langue et en mathématiques, standardisés à l'échelle internationale, administrés à des élèves des troisième et quatrième années du cycle primaire ${ }^{8}$. Comme il s'agit d'une première étude transversale d'un niveau scolaire, elle ne fournit pas d'évaluation longitudinale des projets ou des choix en matière d'éducation. Il faut espérer, cependant, que des travaux constants dans ce domaine produiront une somme de plus en plus riche d'informations sur les performances du système éducatif, qui constituera à terme une documentation précieuse pour effectuer des analyses sur la rentabilité des investissements.

\section{Conception d'un programme et mesures incitatives}

En Amérique latine (comme dans le monde entier), on note un intérêt certain pour l'introduction dans le secteur éducatif de diverses mesures incitatives, par le biais d'une rémunération au mérite, de l'attribution de primes ou d'autres interventions. Cet intérêt, cependant, s'accompagne d'une méconnaissance des expériences et des recherches effectuées dans les autres parties du monde, qui devraient nous mettre en garde contre l'introduction d'un tel système. Les concepteurs de réformes et même les économistes ont tendance à ignorer les débats passionnés et parfois âpres concernant les diverses mesures incitatives et la façon dont elles sont mises en place. Des articles importants, telle l'étude 
de Murnane et Cohen ${ }^{9}$ sur la rétribution au mérite en 1986, restent grandement méconnus.

Au Chili, un exemple d'utilisation de l'analyse économique pour élaborer une politique éducative est donné par le Sistema National de Evaluación del Desempeño Docente (SNED), qui évite certains des problèmes identifiés par Murnane et Cohen en récompensant le mérite des établissements scolaires plutôt que celui de certains enseignants ${ }^{10}$. Aujourd'hui dans sa troisième année de fonctionnement, le système du SNED tire profit de facteurs propres au système éducatif chilien. L'un des plus importants est le processus d'évaluation SIMCE utilisé depuis les années quatre-vingt et qui a déjà fourni des indications sur les connaissances acquises par les élèves. Si le SIMCE n'avait pas existé auparavant, le coût du programme aurait été si élevé que le système SNED n'aurait probablement jamais pu être réalisé. En Amérique latine, très peu de systèmes éducatifs ont mis en place une procédure d'évaluation comparable.

Le principe qui consiste à récompenser les établissements scolaires obtenant les meilleurs résultats est concrétisé par une loi chilienne de $1995^{11}$, qui spécifie entre autres choses six critères de performance: l'efficacité de l'enseignement (mesurée par la procédure SIMCE) ; la progression des résultats (comparaison longitudinale des scores SIMCE); l'initiative (capacité à incorporer les innovations et à tirer parti des aides extérieures); l'amélioration des conditions de travail ; "l'égalité des chances " données aux élèves, mesurée d'après le nombre d'élèves que l'établissement conserve en ses murs et l'intégration des parents et des enseignants dans les initiatives visant à améliorer le fonctionnement de l'établissement. Les données concernant les deux premiers critères sont issus de la procédure SIMCE elle-même, alors que celles propres aux quatre suivants proviennent d'un questionnaire envoyé à chaque établissement et traité en même temps que les tests SIMCE. On combine les informations fournies par ces deux sources pour déterminer un indice de performance.

Pour préserver une certaine équité, les établissements sont répartis entre plusieurs catégories, afin de mettre en compétition des écoles possédant des chances relativement égales de parvenir à de bons résultats. Des récompenses sous forme de subsides sont décernées, dans chaque catégorie, aux établissements ayant obtenu le meilleur indice de performance. Le barème attribuant plus ou moins d'importance aux différents critères fut critiqué pendant les premières années de fonctionnement du SNED, et il a été modifié depuis. Ce barème privilégie les critères d'« efficacité » et de « progression » (à l'origine, $70 \%$ du total, ramenés à $65 \%$ en 1998-1999); celui de l'«égalité des chances », le troisième par ordre d'importance, comptant à l'origine pour $12 \%$ du total, fut porté à $22 \%$ en 1998-1999.

Dans le système chilien de récompenses décernées par le gouvernement central, les primes au mérite peuvent être attribuées à tous les établissements fonctionnant à l'aide de fonds publics, que leur gestion soit municipale ou privée. Les primes sont versées à chaque établissement lauréat puis divisées entre les enseignants, mais $10 \%$ du total sont réservés à ceux d'entre eux qui ont le plus contribué à l'amélioration des résultats. Ceci a pour but de décourager les comportements individualistes. Le montant de la prime est proportionnel à l'effectif de l'établissement. Les récompenses ne sont pas considérables, afin de ne pas provoquer de distorsions et d'éviter les agissements opportunistes. Même si les sommes attribuées restent modestes, il semble que les enseignants les apprécient parce qu'elles ont à leurs yeux la valeur d'un témoignage de satisfaction. 
31 Une évaluation du SNED a montré que ce système jouit de l'approbation générale, en partie parce qu'il procure une source de fonds supplémentaire. Même si le SNED ne fonctionne pas depuis assez longtemps pour qu'on puisse déterminer s'il amène effectivement une amélioration des performances, les observations effectuées dans les établissements indiquent qu'il a un effet positif. Certaines propositions recueillies au cours de l'évaluation méritent d'être citées et concernent: la transparence accrue du système, afin que les établissements comprennent mieux pourquoi les lauréats reçoivent des récompenses; la décentralisation du processus, pour permettre à des instances hiérarchiquement moins élevées de participer davantage aux sélections et de faire entendre leur opinion; la circulation plus large des informations concernant les établissements primés (ce qui donnerait plus de valeur encore au "témoignage de satisfaction » que représentent les récompenses) ; la modification du barème utilisé, pour $\mathrm{y}$ inclure diverses formes de réussite scolaire ; la prise en compte, sans discrimination, des établissements qui acceptent des élèves en très fortes difficultés d'apprentissage.

Bien que le SNED soit un système récent qui n'a pas encore apporté à l'enseignement d'améliorations quantifiables, c'est un exemple intéressant de programme incitatif dont la conception et l'évaluation reposent sur une analyse économique intelligente et bien documentée.

Il y a plusieurs années, Thorsten Husen affirmait que la recherche pédagogique (et j'y ajouterais la recherche économique et l'analyse des orientations) ne détermine pas les politiques éducatives, mais, plutôt, « conditionne le débat » sur les options à prendre. En Amérique latine, les débats ne manquent pas sur les stratégies éducatives, les choix et les réformes et, même si les approches économiques ne sont pas encore un facteur déterminant, elles sont à notre sens de plus en plus prises en compte. On découvre aujourd'hui un nombre croissant d'études et d'analyses économiques pertinentes, subtiles, fondées sur l'observation, bien que la plupart d'entre elles soient effectuées hors des ministères de l'Éducation. Une partie de cette réflexion économique s'infiltre dans les processus d'élaboration des politiques éducatives.

Les éducateurs, les économistes et les politiciens ne parlent pas la même langue et leurs conceptions du monde sont différentes. Les éducateurs ont tendance à ne s'intéresser qu'aux aspects qualitatifs de la transmission et de l'apprentissage des connaissances, alors que les économistes mettent l'accent sur des critères quantifiables, particulièrement les coûts et les résultats. Les politiciens s'intéressent aux résultats uniquement en fonction du bien-être social et de l'approbation de l'opinion publique. Lorsque tous ces acteurs de premier plan de l'élaboration des politiques éducatives pourront débattre à partir de termes et de concepts qui leur seront communs, leurs chances de parvenir à un consensus solide sur des orientations intelligentes et réalisables augmenteront sensiblement. 


\section{NOTES}

1. Robert McMeekin, Education Statistics in Latin America and the Caribbean, Étude financée par la Fondation Ford et réalisée par l'Office régional de l'Unesco pour l'éducation en Amérique latine et aux Caraïbes, Washington, D.C., Inter-American Development Bank, Education Unit, Sustainable Development Department, 1998.

2. Unesco, Santiago, 1996, publié en anglais et en espagnol.

3. OCDE/CERI, Education at a Glance, OECD Indicators 1998, Paris, OCDE, 1998.

4. PREAL, Financiamiento de la Educación en América Latina, Santiago, Chili, PREAL-UNESCO, 1998.

5. Les travaux de George Psacharopoulos à la Banque mondiale ont fait grandement progresser l'utilisation de l'analyse des taux de rendement. Voir : «Returns in Investment in Education : a Global Update ", in World Development, vol. 22, n 9, 1994. Pour l'Amérique latine, voir: Psacharopoulos \& Y.C. Ng. "Earnings and Education in Latin America ", Education Economics, vol. 2, n², 1994.

6. Dans les années quatre-vingt, Paul Hurst et Susie Rodwell de l'Institut d'éducation de l'université de Londres ont mené une étude approfondie des problèmes associés aux changements dans le secteur éducatif et des moyens de les traiter. Un compte rendu de leurs conclusions et des articles consacrés à ce sujet fut publié en 1983.

7. Malcolm Bale \& Tony Dale, «Public Sector Reform in New Zealand and its Relevance to Developing Countries ", World Bank Research Observer, vol. 13, n 1, février 1998, p. 103-22. Voir aussi Peter Aucoin, "Administrative Reform in Public Management: Paradigms, Principes, Paradoxes and Pendulums ", Governance, vol. 3, 1990, p. 115-37.

8. Primer Estudio Internacional Comparativo, Santiago, Unesco-Santiago, 1998.

9. 12 Richard J. Murnane, \& David K. Cohen, « Merit Pay and the Evaluation Problem : Why Most Merit Plans Fail and a Few Survive », Harvard Education Review, vol. 56, nº 1, février 1986, p. 1-17.

10. Les informations sur les primes au mérite décernées par le SNED aux établissements scolaires proviennent des sources suivantes :

Ernesto Treviño, «Sistema National de Evaluación del Desempeño Docente de los Establecimientos Subvencionados", in Innovaciones en el uso de la información en la toma de decisiones de política educativa, sous la direction de Ana María Corvalán et E. Treviño, Santiago, Chili, Unesco, mars 1999.

Vivian Heyl, \& Marcela Guzman, Evaluación del Desempeño, SNED, Santiago, Chili, ministère de l'Éducation, juillet 1998.

Alejandra Mizala \& Pilar Romaguera, Evaluación de la Implementación y Resultados del SNED, 1996-97, Santiago, Centro de Economía Aplicada, universidad de Chile, août 1997.

11. Loi $\mathrm{n}^{\circ} 19410$

Revue internationale d'éducation de Sèvres, 22 | 2013 


\section{RÉSUMÉS}

En Amérique latine, la dimension économique commence à être prise en considération dans l'élaboration et l'orientation des politiques éducatives. En témoignent, malgré les difficultés rencontrées, les évolutions qui se dessinent dans un certain nombre de pays de cette région, notamment au Chili.

In Latin America, the economic dimension is beginning to be taken into account in elaborating and directing educational policies. This is shown by the changes which are appearing in a certain number of countries in this region and in particular in Chile, despite the difficulties encountered.

En América latina, se empieza a tener en cuenta el enfoque económico para la elaboración y la orientación de las politicas educativas. Testigo de ello, a pesar de las dificultades encontradas, las evoluciones que aparecen en varios países de estas zonas, en particular en Chile.

\section{INDEX}

Index géographique : Chili

Mots-clés : économie de l'éducation, financement, politique éducative, économie

\section{AUTEURS}

\section{ROBERT W. MCMEEKIN}

Senior Associate, Centro de Investigación y Desarollo de la Educación, consultant à l'office régional de l'Unesco pour l'éducation en Amérique latine et aux Caraïbes, Santiago, Chili 